\title{
Chinese Adolescents' Moral Reasoning of Rights Attitudes and Psychological Well-Being
}

\author{
Shaogang Yang \\ (Guangdong University of Foreign Studies, 200611497@oamail.gdufs.edu.cn) \\ Sharon To and Charles C. Helwig \\ (University of Toronto, sharonto@gmail.com, helwig@psych.utoronto.ca)
}

\section{Introduction}

In the last twenty years there has been a great expansion of research examining children's attitudes and reasoning about rights, including the rights of children themselves. Initial research, conducted in North America and Europe, revealed that children and adolescents develop conceptions of their own rights in a variety of areas (e.g., Cherney \& Perry 1996; Helwig 1997; Ruck, Abramovitch, \& Keating 1998). Following Rogers and Wrightsman (1978), a conceptual distinction has been made between self-determination and nurturance rights. Self-determination rights pertain to children's freedoms to exercise their agency or choice in a variety of areas of their lives, including choosing one's friends or recreational activities, participation in decision making over issues affecting children, and respect for the child's privacy. In contrast, nurturance rights pertain to the obligations of authorities (e.g., society or parents) to provide for or safeguard the child's emotional, psychological, or physical welfare, and include, as examples, protection from abuse or harm and the provision of emotional support, education, and adequate health care. In general, this research has found that both children and adolescents tend to endorse children's nurturance rights, and that support for self-determination rights increases across adolescence in accordance with beliefs about children's developing autonomy and competence (Helwig 2006; Ruck et al. 1998).

Over the last decade especially, research on children's rights has expanded to include other, non-Western cultural contexts. For example, endorsements of various types of rights pertaining to both freedoms and welfare have been found among children and adolescents in China (Lahat, Helwig, Yang, Tan, \& Liu 2009), Malaysia (Cherney \& Shing 2008), Africa (Day 2014; Ruck, Tenenbaum, \& Willenberg 2011), and in Muslim populations living in the Middle East (e.g., Ben Arieh \& KhouryKassabri 2008). This research has shown that concepts of freedoms and rights are not simply tied to Western cultural systems but are endorsed, with some variations, by children in a variety of cultures around the world.

Despite this widening interest in children's rights by developmental psychologists, research on the factors that facilitate the development of rights understandings remains highly limited. Although some research has begun to examine the correlates of attitudes and reasoning about children's rights in North America (e.g., Peterson-Badali, Morine, Ruck, \& Slonim 2004), including the role played by various aspects of parenting styles such as authoritative or authoritarian parenting, virtually nothing is known about the factors that contribute to the development of rights understandings in nonWestern cultural contexts. Moreover, although several prominent philosophers (e.g., Nussbaum 2000; Sen 2009) have argued that the provision of rights to welfare and self-determination may contribute to human flourishing and psychological well-being in diverse cultural settings, this issue has not been directly addressed in developmental research on children's rights. For example, little is known about whether the features of environments that support the 
development of rights understandings also may contribute to children's psychological well-being. This question may be especially important to address in non-Western and more traditional cultural environments (e.g., urban and rural Mainland China) where children's rights - especially those pertaining to self-determination and autonomy - may not be emphasized as part of the cultural tradition (Naftali 2009). Accordingly, the present study examined relations between several factors theorized to contribute to the development of notions of children's rights, such as parental and teacher autonomy support and responsiveness, and democratic family and school climate, and their associations with adolescents' psychological well-being, in a sample of adolescents from urban and rural China.

\section{Correlates of Rights and Psychological Well-Being}

An additional question is what role do children's self-determination and nurturance rights, and the features of socialization environments that facilitate or hinder these constructions, play in children's psychological well-being? Documents such as the UN Convention on the Rights of the Child have affirmed the recognition of children's rights to both nurturance and self-determination as important political avenues for securing children's physical and psychological well-being (United Nations General Assembly, 1989). Yet, we are aware of no research that has directly explored these potential connections. However, there is an emerging body of research on relations between children's psychological well-being and some of the proposed socialization correlates of children's rights, such as school and family autonomy support and responsiveness and children's own participation in decision making. For example, several studies have revealed that both parental and teacher support for children's autonomy is related to improved psychological well-being, measured variously in terms of fewer symptoms of psychopathology (e.g., anxiety or depression) or positively in terms of heightened subjective well-being and self-esteem (e.g., Chirkov \& Ryan 2001; Joussemet, Mageau, \& Koestner 2014). Also, perceived parental or teacher responsiveness (caring) has been found to predict less psychological distress among adolescents (Bogenschneider \& Pallock 2008; Wentzel 1997). Similar positive relations have been found in an emerging research literature on perceptions of democratic or autonomy supportive school climate (Vieno, Perkin, Smith, \& Santinello 2005; Way, Reddy, \& Rhodes 2007). For example, in a study of over 1400 American adolescents, Way et al. (2007) found that a school climate in which student autonomy was supported through involvement in classroom decisions predicted fewer adolescent psychological and behavioral problems and higher levels of self-esteem.

However, most of this research has been conducted with samples from Western countries (i.e., in North America and Europe); research on these issues has only begun to be conducted in non-Western cultural settings, including China. Positive relations between parental autonomy support and psychological well-being have been found for adult mainland Chinese students (Vansteenkiste, Zhou, Lens, \& Soenens 2005), and school democratic climate has been linked to psychological health and higher self-esteem among Chinese urban adolescents (Jia et al. 2009). Furthermore, among adolescents in Japan and the United States, perceived parental intrusion into adolescents' sphere of autonomy over personal issues (e.g., choice of recreational activities or friends) was found to be associated with internalizing disorder symptoms, such as anxiety and depression (Hasebe, Nucci, \& Nucci 2004).

Existing research on this topic, however, is limited in several respects. First, all of the research, including the few studies conducted in non-Western settings such as China, has involved urban samples, which may be argued to be more modern and hence possibly influenced by Western ideologies or practices (Greenfield 2009). Second, although all of these studies have generally found positive relations between these 
various dimensions (e.g., autonomy support, responsiveness, democratic school and family climate) and psychological well-being, the relative contribution of each of these factors is not clear, as most studies have only focused on one of these dimensions. As well, with the exception of Chirkov and Ryan (2001), most studies have measured variables in only one social context (family or school); thus little is known about the relative contributions of the family or school environment to children's psychological well-being, and whether similar patterns and relations among these variables holds across these contexts.

\subsection{The present study}

The present study examined both urban and rural Chinese adolescents' attitudes towards children's rights, and their perceptions of parental and teacher autonomy support and responsiveness, democratic school and family climate, and psychological well-being. Our study incorporated samples from both urban and rural China, in order to extend investigation of these issues into understudied and more traditional rural populations. The rural/urban distinction is particularly relevant in China, given the vast differences in modernization, economic standard of living, parenting practices, and traditional values uncovered in prior investigations comparing urban and rural Chinese settings (e.g., Helwig et al. 2014; Zhang \& Fuligni 2006; Zhang, Zheng, \& Wang 2003). Thus, exploring children's attitudes toward their own rights and their hypothesized correlates in more traditional, rural settings like that found in China may provide a stronger test of their universality than prior work using samples drawn from Western societies or modern, urban environments, where children's rights and autonomy is given more emphasis (Naftali 2009).

Based on prior research in diverse cultural settings, we predicted that children's rights would be endorsed by Chinese adolescents from both urban and rural settings. However, we also expected that children's self-determination rights would be endorsed more strongly by urban than rural adolescents, in accordance with the influence of factors such as modernization, socioeconomic status, and urban setting on children's support for self-determination and autonomy found in prior research (e.g., Melton 1980; Lahat et al. 2009; Nucci et al. 1996). At the same time, we also predicted that support for children's self-determination rights would increase with age, in accordance with universal processes governing the development of autonomy in adolescence hypothesized to exist across cultures and reflected in findings from prior research (Erikson 1968; Helwig 2006, Nucci 2001).

Based on our prior overview of existing research, we expected that parent and teacher responsiveness would predict both types of rights, whereas support for self-determination rights would be primarily associated with perceptions of autonomy support. In addition, we expected that perceptions of home and school tolerance of dissent would be an especially strong predictor of support for children's self-determination rights, based on prior research (Khoury-Kassabri \& Ben Arieh 2009). In our investigation and analysis strategy, we were attuned to the potential for both general positive relations among these variables as well as more reactive or "oppositional" pathways that might result when perceived autonomy support and responsiveness were particularly low (Lo et al. 2011, Vansteenkiste et al. 2014). Based on the prior research conducted in various cultural settings reviewed earlier, we expected that both perceived parental and teacher autonomy support and responsiveness would be positively associated with adolescents' psychological well-being.

We also sought to examine the unique contribution made by democratic school and family climate to children's attitudes toward their rights and their psychological well-being. Based on prior psychological research on democratic climate (e.g., Khoury-Kassabri \& Ben-Arieh 2009; Vieno et al. 2005) as well as general democratic 
theorizing (Cohen 1971; Richardson 2002), we devised our own measure comprising several key features of democratic climate applicable to family and school settings involving children, such as children's involvement in decision making, respect for their freedom of expression, and children's rights to due process (e.g., having their perspective considered when accused of wrongdoing). Although the manner of conceptualizing and measuring autonomy within Self Determination Theory (e.g., Chirkov \& Ryan 2001; Robbins 1995) includes some of these dimensions (e.g., respect for the child's opinions and input), it also includes other facets of autonomy that are not necessarily related to democratic conceptions (e.g., encouraging the child to explore their interests or to develop their individuality). Some cultural psychologists have argued that self-expression and individuality are culturally more Western forms of autonomy that may not be encouraged or valued as highly in other cultures, such as Asian societies (e.g., Markus \& Kitiyama 1991). Thus, we sought to distinguish more purely democratic aspects of autonomy from the more general and heterogeneous conception incorporated in the SDT measures in order to examine the unique contribution that perceptions of democratic school and family climates may make to Chinese adolescents' understandings of their rights and to their psychological well-being. Based on the prior work reviewed earlier, we expected that perceptions of democratic climate would be positively related to both adolescents' support for self-determination rights and their reported psychological well-being.

\section{Method}

\subsection{Participants and Research Sites}

The total sample consisted of 395 Chinese adolescents from two age groups drawn from two research sites representing urban and rural China. Among them, 195 participants were from the city of Guangzhou (13- to 15-year-olds, $\mathrm{n}=92,45$ males, $M=$ $13.90, S D=.78$; 16 - to 19-year-olds, $\mathrm{n}=103$, 37 males, $M=16.99, S D=.82$ ) and 200 participants were from a rural village in Northern Guangdong Province (13- to 15 -year-olds, $\mathrm{n}=96,52$ males, $M=14.51, S D=.82 ; 16$ - to 19 -year-olds, $\mathrm{n}=104,53$ males, $M=16.81, S D=.81$ ). These research sites were selected to provide a contrast between adolescents who were from a modern, economically-developed city and those who were from a much more traditional, agriculturally-based, rural community with far less exposure to Western influences.

The urban Chinese subsample was drawn from schools serving a largely working to middle-class population located in the city of Guangzhou, the capital and largest city of Guangdong province. Guangzhou is a modern city and one of the key commercial and trading centers of China. Residents of Guangzhou, like those in other modern cities in China, overall have a much higher standard of living, level of education, and exposure to foreign cultures than people living in rural areas. Average per capita wage for residents of Guangzhou is 30,658 yuan, or US\$4,977 (Guangdong Statistical Yearbook, 2010). In terms of occupation and education level, 19\% of Chinese urban participants' parents were employed in professional, sales, managerial, or other technical occupations, $28 \%$ were employed in service occupations, trades, or manufacturing, $26 \%$ were employed in family-run businesses, and 22\% (mostly mothers) were homemakers or unemployed, with $5 \%$ of unknown occupation. Nine percent had completed university or had some post-secondary education, $75 \%$ had completed high school only, and $14 \%$ had completed grade school only, with $2 \%$ of unknown education level.

The rural Chinese subsample was drawn from schools located in a more remote area of Renhua County in Northern Guangdong Province. The region from which the sample was drawn was chosen to be representative of a typical rural and agricultural region in China, and thus substantially less developed economically and more traditional in character than the larger urban centers. Average family household income for the 
district from which the sample was drawn was 6,317 yuan, or approximately US $\$ 1,025$ (Guangdong Statistical Yearbook, 2010). The majority (55\%) of parents of Chinese rural participants were farmers by occupation, with the remainder working in local industry $(12 \%)$ or running small businesses in the area (13\%) or of unknown occupation $(20 \%)$. Two percent of parents had completed university or had some post-secondary education, $14 \%$ had completed senior high school, and most had only completed junior high school (49\%) or grade school (28\%). One percent of parents had never received any education and six percent did not report their parental education level.

\subsection{Measures}

Several self-report questionnaires were used in this study. Chinese translations of questionnaires were pilot tested with urban and rural Chinese adolescents in focus groups and wordings were modified as necessary to improve readability and comprehension. Any question items that were deemed culturally inappropriate were replaced (see below) with more suitable items. All final translated Chinese questionnaires were then back translated into English by a different native Chinese bilingual translator to ensure the accuracy of the translations.

\subsubsection{Children's rights attitudes}

An adaptation of the Children's Rights Attitude (CRA) questionnaire (Peterson-Badali et al. 2003, originally derived from Rogers \& Wrightsman 1978) was used to measure Chinese adolescents' attitudes toward children's rights issues. Autonomy support and responsiveness. Participants were given the Perception of Parents (POPS) scales (originally derived from Robbins 1995) and a corresponding measure for teachers (see Chirkov \& Ryan 2001) to assess their perceived autonomy support and responsiveness (warmth and involvement) in family and school settings. In accordance with calls to examine the unique role of mothers and fathers in socialization (e.g., Marsiglio, Amato, Day, \& Lamb 2000), the dimensions of autonomy support and responsiveness were assessed separately for mothers and fathers in this study. The internal consistency for the parent and teacher autonomy support and responsiveness subscales for the present sample was acceptable (Cronbach's alpha for autonomy support $=.68$ (mother), .73 (father), .70 (teacher); and for responsiveness $=.83$ (mother), .87 (father), .84 (teacher)).

\subsubsection{Democratic climate}

In order to examine the unique role of democratic school and family environments, a new measure of democratic climate was devised based on prior research (e.g., Khoury-Kassabri \& Ben-Arieh 2009).

\subsubsection{Psychological well-being}

Finally, participants completed two measures of psychological well-being. Given the hypothesized relations between parental and teacher control of autonomy and internalizing symptomology (e.g., Chirkov \& Ryan 2001; Hasebe et al. 2004), participants were given the 10-item version of the Children's Depression Inventory (CDI short; Kovas 1980).

\section{Results}

\subsection{Preliminary exploratory analysis of reactive patterns}

Given that prior research on rights concepts (e.g., Day et al. 2006) has sometimes yielded findings suggesting the existence of reactive patterns, in which adolescents displayed a heightened level of self-determination rights endorsement when perceived autonomy support and responsiveness were low, as a first step in the analysis, we explored our data for such patterns. Cases meeting both of the following observations 
were identified as potential reactive cases for exploratory analyses: (i) those falling one standard deviation below sample mean for at least two of the family and school predictor variables (i.e., maternal, paternal, or teacher autonomy support; maternal, paternal, or teacher responsiveness; and home or school democratic climate), in combination with (ii) endorsement of self-determination rights that is at least one standard deviation above sample mean. Eighteen cases met these criteria: Twelve from the urban sample and six from the rural sample. Table 1a displays the means, standard deviations, and zero-order correlations for the full sample. With the reactive cases included in the analyses, we found several such reactive patterns indicating negative relationships between support for self-determination rights and mother responsiveness $(r=-.14, p<.01)$, father responsiveness $(r=-.13, p<.05)$, and home democratic climate $(r=-.15, p<.01)$. Moreover, support for self-determination rights was significantly positively correlated with the level of depression $(r=.14, p<.01)$. When these reactive cases were examined separately, significant negative associations between support for self-determination rights and teacher responsiveness $(r=-.70, p<.01)$ and teacher autonomy support $(r=-.64, p<.01$ ) were found, along with negative (but not significant) associations for all of the other home and school predictor variables. However, in subsequent analyses when the reactive cases were removed from the analysis, all of these unexpected significant pairwise correlations found in the full sample (i.e., the significant negative relations between endorsement of self-determination rights and mother responsiveness, father responsiveness, home democratic climate, and depression) were no longer significant (see Table 1b). Thus, all subsequent analyses were conducted with the eighteen reactive cases removed.

\subsection{Analysis plan for main sample}

In the following analyses, t-tests and MANOVAs were performed to test for mean differences of the outcome variables (i.e., adolescents' attitudes toward children's rights and their psychological well-being) by gender, setting, and age group. Zero-order correlations among all study variables are reported next, followed by four hierarchical regression analyses, for nurturance rights attitudes, self-determination rights attitudes, and self-reported life satisfaction and depression, respectively, to test if and how the various parental and teacher factors predicted the outcome variables. In the regression analyses for children's rights attitudes, support for nurturance rights and self-determination rights were regressed on five blocks of independent variables separately. All non-dichotomous variables were centered around their means by subtracting each mean from each variable prior to analyses to reduce problems with multicollinearity (Aitken \& West 1991). Dummy variables were created for gender $(1=$ male), age group ( $1=13$ - to 15 -year-olds), and setting $(1=$ urban $)$ and were entered at the first step in the model to control for immediate effects of demographic variables on children's rights attitudes. To assess the effects of features of socialization environment on children's rights attitudes, adolescents' perceived maternal, paternal, and teacher support for autonomy and responsiveness were entered at the second step, followed by measures of democratic home and school climate at the third step, and tolerance of dissent at home and school at the fourth step. We entered a block of eight interaction terms that crossed setting with each of the home and school environment variables at the fifth step to test for any moderating effects of setting. Variables in blocks that do not contribute significantly to the regression model (at the .05 level) were eliminated from the final regression model.

\subsection{Descriptive and correlational analysis}

Means and standard deviations of all study variables are presented in Table 1, for (b) the full sample without reactive cases, (c) the urban subsample, and (d) the rural subsample. As indicated by the means, rural adolescents perceived less tolerance of dissent at home compared to their urban counterparts, $t(374)=3.40, p<.005, d$ 
$=.35$. Participants' support for children's rights and their reports of psychological well-being were analyzed with 2 (gender) X 2 (age group) X 2 (setting) ANOVAs. Analyses for children's rights attitudes revealed several significant age group and setting main effects. As expected, both older $\left(F(1,335)=4.32, p=<.05, \quad \eta^{2}=.01\right)$ and urban $\left(F(1,335)=14.09, p=<.001, \quad \eta^{2}=.04\right)$ adolescents endorsed higher levels of self-determination rights. Somewhat unexpectedly, older $(F(1,335)=6.88, p$ $\left.=<.01, \quad \eta^{2}=.02\right)$ and urban $\left(F(1,335)=27.25, p=<.001, \quad \eta^{2}=.08\right)$ adolescents also endorsed higher levels of nurturance rights. Regarding measures of psychological well-being, adolescents from the urban setting reported a higher level of life satisfaction compared to their counterparts in the rural area, $F(1,326)=31.67, p$ $<.001, \quad \eta^{2}=.09$.

Table $1 \mathrm{~b}$ displays bivariate correlations among study variables for our sample. As expected, all of the home and school environment variables were generally correlated with one another. Specifically, adolescents' perceived parental and teacher autonomy support were correlated with reports of their responsiveness $(r=.66$ to $.74, p<.001)$. Moreover, parental support for autonomy and responsiveness were both correlated with reports of democratic climate at home $(r=.51$ to $.55, p<.001)$, and teacher support for autonomy and responsiveness were both correlated with democratic climate at school $(r=.64, p<.001)$. Tolerance of dissent at home and school, however, was only correlated with parental and teacher autonomy support, respectively $(r=.11$ to $.17, p<.05)$, as well as democratic home and school climate $(r$ $=.13$ to $.19, p<.01)$, but not with any of the corresponding responsiveness measures. Also as expected from prior research in Western cultural settings, adolescents' attitudes towards nurturance rights were positively related to their attitudes towards self-determination rights $(r=.34, p<.001)$.

In the present study, we hypothesized that support for nurturance rights would be associated with parent and teacher autonomy support and responsiveness and democratic home and school climate. These hypothesized relations were generally supported. Specifically, support for nurturance rights was positively associated with parental and teacher autonomy support, maternal responsiveness, and democratic home and school climate (Table 1b). It was further expected that support for self-determination rights would be associated mainly with home and school autonomy support, democratic home and school climate, and tolerance of dissent. As Table $1 \mathrm{~b}$ indicates, support for self-determination rights was positively associated with tolerance of dissent at home and at school, but did not correlate with any other family and school environment variables. Lastly, our expectations regarding relations between home and school variables and psychological well-being were generally supported. Both life satisfaction and depression (reversed) were positively related to all of the home and school environment variables except for tolerance of dissent at home and at school.

\subsection{Hierarchical regression analyses: Predictors of children's rights attitudes and} adolescents' psychological well-being

To further assess how home and school variables contribute to adolescents' attitudes towards children rights, we performed two hierarchical multiple regression analyses on nurturance and self-determination rights attitudes, respectively. A multiple step model was employed to examine the unique contribution of different family and school environmental variables while controlling for background factors. Whereas some of the predictor variables were not significantly related to outcome variables at the 
bivariate level, to accurately assess the contribution of each independent variable to the outcome, all theoretically relevant independent variables were retained and included in the model (Pandey \& Elliott 2010).

\subsubsection{Nurturance rights}

The regression results are presented in Table 2. Consistent with the results of the ANOVA, significant main effects were found for age and setting, which accounted for $12 \%$ of the variance in support for nurturance rights. Adolescents from the urban setting and those from the older age group endorsed a higher level of nurturance rights. Predictor variables in block 2, consisting of main effects of parental and teacher support for autonomy and responsiveness, accounted for an additional $6 \%$ of the variance in the regression analysis. Maternal responsiveness and teacher autonomy support significantly predicted higher levels of endorsement of nurturance rights. Unexpectedly, teacher responsiveness predicted lower support for nurturance rights. Upon further inspection, a suppression effect with teacher responsiveness serving as the suppressor variable was found (Cohen, Cohen, West, \& Aiken 2013). While teacher responsiveness was uncorrelated with support for nurturance rights, $r(359)=.06, p=.30$, it contributed significantly to the model by suppressing irrelevant variance in the measure of teacher autonomy support. The negative regression weight of teacher responsiveness is a result of the suppressor's low correlation with the outcome variable (support for nurturance rights) and high correlation with the other predictor variable (teacher autonomy support) at the bivariate level. Analyses revealed that this suppressor effect was not the result of high levels of multicollinearity between teacher responsiveness and any other predictors. The third, fourth, and fifth block of regression, which consisted of the home and school democratic climate, tolerance of dissent, and interaction effects respectively, did not account for additional variance and hence were eliminated from the model. Together, the regression model accounted for $18 \%$ of the variance in support for nurturance rights.

\subsubsection{Self-determination rights}

Within the first block of control variables, significant main effects were found only for setting, explaining $8 \%$ of the variance in support for self-determination rights. After controlling for the demographic variables in the second block, maternal autonomy support was found to be the only significant predictor in this step, accounting for an additional $4 \%$ of the variance. The introduction of democratic climate variables in the third block did not contribute significantly to the regression model and hence the variables within the block were not further investigated. In the fourth step of the regression analyses, tolerance of dissent at home was found to be a significant predictor in the positive direction, predicting attitudes of self-determination rights above and beyond what was predicted by more general measures of parental and teacher autonomy support and responsiveness, as well as democratic home and school climate. Lastly, the results showed that the inclusion of interaction terms did not significantly explain any additional variance in support for self-determination rights and hence were dropped from the model. Together, the regression model accounted for $17 \%$ of the variance in self-determination rights attitudes.

\subsubsection{Adolescents' psychological well-being}

To assess whether various features of family and school environment predicted adolescents' psychological well-being, two hierarchical multiple regression analyses were performed with life satisfaction and depression (reversed), respectively (see Table 3 ). When demographic variables were entered in the first block, urban setting was associated with higher life satisfaction. Controlling for demographic variables, teacher responsiveness significantly predicted adolescents' level of depression in the second block. Adolescents who perceived higher levels of teacher responsiveness reported 
lower levels of depressive symptoms. Home democratic climate was a significant predictor of both life satisfaction and depression level in block 3, with adolescents who reported more democratic family climates reporting higher life-satisfaction and fewer depressive symptoms. Thus, democratic climate appears to make a unique contribution to adolescents' psychological well-being, above and beyond what was predicted by the general measures of autonomy support and responsiveness. Neither the addition of tolerance of dissent nor interaction parameters in the fourth and fifth step improved the predictive power of the model and hence were removed from the final model. Together, the predictor variables accounted for $30 \%$ of the variance in adolescents' life satisfaction scores and $21 \%$ of their depression scores.

\section{Discussion}

This study examined urban and rural Chinese adolescents' attitudes toward children's rights, and how these attitudes relate to several dimensions of socialization in their family and school environments, including perceptions of parental and teacher autonomy support and responsiveness and family and school democratic climate. Relations between these dimensions of socialization and adolescents' psychological health and well-being also were examined. This study is the first to systematically investigate these correlates of children's rights attitudes and psychological well-being in a non-Western culture and in a traditional, rural setting. The findings provide support for several theoretical propositions derived from Self-Determination Theory (Deci \& Ryan 2013) and democratic socialization research (e.g., Vieno et al. 2005) regarding relations between psychological well-being and patterns of socialization in family and school contexts.

In accordance with prior research revealing age-related increases in adolescents' striving for autonomy within different cultural contexts (Helwig 2006; Smetana 2011), we found that support for children's self-determination rights increased with age. As well, reasoning about both types of rights was influenced by urban versus rural setting, with urban Chinese adolescents endorsing children's rights more strongly. This finding probably reflects the increasing attention paid to children's rights in modern, urban Chinese settings, where higher standards of living and parental education levels mean that children's needs and autonomy often receive greater attention than in more traditional, rural settings (Naftali 2009). Interestingly, and unexpectedly, we also found that support for nurturance rights increased with age across both urban and rural samples. This finding may reflect the relative underemphasis in China on children's rights of all types. For example, parental shaming and corporal punishment of children are more common in China than in Western cultural settings (Helwig et al. 2014; Naftali 2009) and may lead to diminished expectations regarding children's claims to some nurturance rights. Thus, in addition to expansions in the scope of Chinese children's autonomy during adolescence, there may be further changes during this age period in their understandings of the obligations of authorities to provide for children's physical protection and psychological welfare.

Several predictions about the correlates of children's rights attitudes derived from prior work in Western or other cultural settings (e.g., Khouri-Kassabri \& Ben-Arieh 2009; Peterson-Badali et al. 2004) were supported. We found that children's nurturance rights followed a straightforward pattern in which positive attitudes toward these rights were facilitated by responsive, autonomy supportive, and democratic family and school environments. Maternal responsiveness and teacher autonomy support were especially important, as indicated by the finding that only these variables remained significant predictors of children's nurturance rights attitudes after the other variables were controlled for in the regression.

We hypothesized that endorsements of children's self-determination rights would be facilitated by parental and teacher support for children's autonomy and by 
democratic home and school environments that encourage children's input into decisions and that respect their basic freedoms. Although we did not find that democratic climate was related to support for self-determination rights, maternal autonomy support did predict higher levels of endorsement of self-determination rights when other variables were controlled in the regression, as found in research in North American cultural settings (e.g., Peterson-Badali et al. 2004). These findings provide tentative support for the proposition that socialization agents who encourage and support children's autonomy may facilitate positive attitudes toward children's self-determination rights across diverse cultural settings. At the same time, we also explored the existence of an "oppositional" pathway that may enhance children's awareness of and support for self-determination when basic needs for autonomy are not perceived as being met (Lo et al. 2011; Vansteenkiste et al. 2014). In the present study, we found evidence of such an "oppositional" pathway in the form of significant negative associations between support for self-determination rights and socialization predictors such as maternal and father responsiveness and home democratic climate, as well as negative relations between support for self-determination rights and adolescent psychological well-being. These significant negative associations were entirely due to the contribution of a small number of participants who showed very high levels of support for self-determination rights and very low levels of perceived autonomy support, responsiveness, and democratic climate in the family and school. When these participants were removed from the analysis, these unexpected negative correlations became non-significant. This finding highlights the importance of accounting for reactive or oppositional pathways, as well as more straightforward or facilitative relations, in research on the socialization correlates of children's rights attitudes, and may help to explain some contradictory findings of prior research (e.g., Day et al. 2006; Peterson-Badali et al. 2004).

Also as predicted, support for self-determination rights was found to be positively correlated with tolerance of dissent in the home and school. The latter finding is consistent with prior research showing strong associations between tolerance of dissent and support for children's rights among Israeli and traditional Arab adolescents residing in Jerusalem (Khoury-Kassabri \& Ben-Arieh 2009). Evidence of this association across such diverse cultural settings (i.e., among different ethnicities in the Middle East and now in urban and rural China) strongly suggests that universal processes may be at work. It is reasonable to suppose that family and school environments that encourage the expression of dissent and criticism may enhance adolescents' sense of their own autonomy, leading to greater support for children's self-determination rights. However, the opposite causal relationship also may account for these patterns, i.e., it may be that those adolescents who initially have a greater awareness of children's self-determination rights may be more empowered to assert their autonomy and thereby influence their family or school environments to become more tolerant of dissent. As argued by many current theorists (e.g., Kuczynski \& Knafo 2014; Smetana 2011), socialization is a reciprocal process, and it may be reasonable to expect bidirectional influences here. Further research, preferably of a longitudinal nature, is needed to tease out the precise nature of these causal relationships.

The results of our study provide important confirmation, in a non-Western and highly traditional cultural environment, of several propositions of Self-Determination Theory regarding relations between psychological well-being and autonomy support and responsiveness. Although prior studies have revealed relations between psychological well-being and autonomy support in modern, urban samples in a variety of cultures (e.g., Chirkov \& Ryan 2001), this is the first study we are aware of to show that these relations also hold for adolescents from a highly traditional, rural, non-Western cultural setting. We found that parental and teacher autonomy support and responsiveness were equally highly correlated with psychological well-being (both depression and life-satisfaction) in both our urban and rural samples, supporting the 
theoretical proposition from SDT that the needs supported by these socialization practices (e.g., needs for autonomy and relatedness) are functionally related to psychological well-being in diverse cultural settings (Deci \& Ryan 2013).

The current study however extends SDT theory by investigating the unique contribution of democratic aspects of autonomy support and responsiveness to children's psychological well-being. We found that family environments that are perceived as more democratic, as reflected in recognition of adolescents' freedom of expression, provision for their participation in decision making, and respect for due process and fairness, made unique and positive contributions to adolescents' psychological health, even when general parental autonomy support and responsiveness were controlled in the regression. Our findings suggest that children's needs for autonomy may be optimally satisfied within democratic social structures having these features. These findings are important as they connect basic rights and freedoms, and their support in contexts of socialization such as the family, to healthy psychological functioning and well-being. As such, these findings may be seen as providing empirical support for recent philosophical perspectives highlighting the role of democratic social environments in enhancing human freedoms and capabilities and thereby promoting psychological health (e.g., Nussbaum 2000; Sen 2009). Although sometimes argued to be relevant only to Western societies, our findings instead show that democratic social structures may be an important means for realizing human happiness in diverse cultural settings.

A particular strength of this study was the simultaneous exploration of socialization predictors across social contexts and agents (e.g., parents, teachers). It is perhaps not surprising that maternal autonomy support and responsiveness emerged as important predictors of adolescents' rights attitudes, given mothers' primary role in socialization in China (and in most cultures), but it is noteworthy that teacher responsiveness emerged as the only significant predictor of adolescents' psychological well-being once all of the other variables were controlled for in the regression. This, in conjunction with the contribution of teacher autonomy support noted earlier as a predictor of nurturance rights attitudes, suggests that teachers may play an especially important role in children's socialization and psychological well-being in China. Students in China typically spend long hours in school settings and frequently have close personal relationships with their teachers (Jia et al. 2009). Responsive teachers thus may help offset some of the negative psychological consequences of lack of autonomy support or responsiveness at home, for example. It is also striking that teachers' role, at least as a predictor in our data, was stronger even than that of fathers (none of the correlations involving the father variables were significant in the regression). This may reflect the fact that fathers in China are less involved in child care and are often away, working long hours.

There are several limitations to the present study that should be acknowledged. First, we examined contemporaneous relations among variables such as psychological well-being and various socialization correlates (e.g., autonomy support and responsiveness and democratic climate), with the theoretically-derived assumption that these variables directly contribute to enhanced psychological well-being (Deci \& Ryan 2013). Our findings generally confirmed these expected relations and are consistent with that of prior research in Western cultural settings. However, our cross-sectional design could not definitively establish causality; longitudinal investigations would be needed to better establish the direction of effect and specific causal contributions of each of these variables to adolescents' psychological well-being. Nevertheless, our findings are generally in-line with those of recent longitudinal research on academic motivation showing, for example, that gains in autonomy among Chinese and U.S. adolescents subsequently enhance psychological well-being (e.g., Qin et al. 2009).

Second, consistent with SDT's focus on the psychological experience of need satisfaction, our study relied on adolescents' perceptions of parent and teacher behavior 
and how democratic they perceived their home and school environments to be. Although adolescents' perceptions of these aspects of their school and family environments showed clear relations with psychological well-being, we do not know how these perceptions correspond to objective features of their home and school environments. It may be that home or school environments perceived by Chinese adolescents as highly democratic or autonomy supportive would be seen quite differently by Western adolescents, for example. Future research might include direct observations of different types of family or school environments (e.g., those high or low in autonomy support or democratic climate) to better determine how objective features of socialization contexts affect adolescents' perceptions and their corresponding psychological health.

\section{Conclusions}

Our study showed that adolescents from both modern and traditional settings in a non-Western culture support children's rights to self-determination and nurturance. Attitudes toward different types of rights were differentially associated with various features of socialization contexts. Parental and teacher autonomy support and responsiveness were most associated with support for children's nurturance rights, whereas perceived tolerance of dissent and maternal autonomy support were most related to support for children's self-determination rights. Our findings further show that family and school environments perceived as supportive of adolescents' autonomy and responsive to their needs are associated with improved psychological health and functioning, consistent with theoretical perspectives that identify autonomy as a basic psychological need (Deci \& Ryan 2013; Hasebe et al. 2004). At the same time, our study also illuminated the specific role played by democratic climate in adolescents' psychological functioning. Our findings contribute to a growing body of research that suggests that environments that are structured more democratically and that are responsive to individuals' autonomy needs contribute to their psychological health and well-being across diverse cultural settings. To conclude:

Our research has got the following conclusions:

(1) Chinese Adolescents assert both nurturance and self-determination rights.

(2) Self-determination and nurturance rights become more salient with increasing age in both rural and urban contexts.

(3) Perceived personal autonomy and democratic climate positively related to psychological well-being.

(4) Concepts of rights and freedoms have their source in universal needs for autonomy and developmental processes, rather than culturally-transmitted orientations (e.g., individualism and collectivism).

Despite the fact that these adolescents came from mainland China, a country often described as highly collectivistic and less supportive of freedoms, individual rights, and democratic autonomy than Western societies, our findings were true even for much more traditional rural populations within Mainland China, so these findings cannot be explained in terms of urbanization or cosmopolitanism or globalization. More broadly, our findings suggest that concepts of rights and freedoms may have their source in universal needs for autonomy, and in how these needs intersect with developmental processes, rather than in purely culturally-defined and transmitted moral orientations.

\section{Literature}

Aiken, L. S. \& West, S. G. 1991. Multiple Regression: Testing and Interpreting Interactions. Newbury Park: Sage. 
Baumrind, D. 1978. Parental Disciplinary Patterns and Social Competence in Children. Youth and Society, 9, 239-276.

Ben-Arieh, A. \& Khoury-Kassabri, M. 2008. Attitudes toward and Understanding of Children's Rights Among Middle School Students in Jerusalem: The Role of Family Values and Patterns, Nationality, and Religion. American Journal of Orthopsychiatry, 78, 359-368.

Bogenschneider, K. \& Pallock, L. 2008. Responsiveness in Parent-child adolescent relationships: Are influences conditional? Does the reporter matter? Journal of Marriage and the Family, 70, 1015-1029.

Cohen, J., Cohen, P., West, S. G., \& Aiken, L. S. 2013. Applied Multiple Regression/Correlation Analysis for the Behavioral Sciences (3rd ed.). New York: Routledge.

Cherney, I. D. \& Perry, N. W. 1996. Children's Attitudes toward Their Rights-An International Perspective. In E. Verhellen (ed.), Monitoring Children's Rights (pp. 241-250). Boston: Martinus Nijhoff.

Cherney, I. D. \& Shing, Y. L. 2008. Children's Nurturance and Self-Determination Rights: A cross-cultural Perspective. Journal of Social Issues, 64, 835-856.

Chirkov, V. \& Ryan, R. M. 2001. Parent and Teacher Autonomy Support in Russian and US Adolescents: Common Effects on Well-Being and Academic Motivation. Journal of Cross-Cultural Psychology, 32, 618-35.

Cohen, C. 1971. Democracy. New York: The Free Press.

Day, D. M., Peterson-Badali, M., \& Ruck, M. D. 2006. The Relationship Between Maternal Attitudes and Young People's Attitudes toward Children's Rights. Journal of Adolescence, 29, 193-207.

Day, K. 2014. The Right to Literacy and Cultural Change: Zulu Adolescents in Post-Apartheid Rural South Africa. Cognitive Development, 29, 81-94.

Deci, E. \& Ryan, R. M. 2013. The Importance of Autonomy for Development and Well-Being. In B. W. Sokol, F. M. E. Grouzet, \& U. Muller (eds) Self-Regulation and Autonomy: Social and Developmental Dimensions of Human Conduct (pp. 19-46). New York: Cambridge University Press.

Dewey, J. 1916. Democracy and Education. New York: MacMillan.

Diener, E., Emmons, R. A., Larsen, R. J., \& Griffen, S. 1985. The Satisfaction with Life Scale. Journal of Personality Assessment, 49, 71-75.

Erikson, E. 1968. Identity, Youth, and Crisis. New York: W. W. Norton.

Greenfield, P. M. 2009. Linking Social Change and Developmental Change: Shifting Pathways of Human Development. Developmental Psychology, 45, 401-18.

Guangdong Statistical Yearbook. 2010. Beijing: China Statistics Press.

Hasebe, Y., Nucci, L., \& Nucci, M. S. 2004. Parental Control of the Personal Domain and Adolescent Symptoms of Psychopathology: A Cross-National Study in the United States and Japan. Child Development, 75, 815-28.

Helwig, C. C. 1997. The Role of Agent and Social Context in Judgments of Freedom of Speech and Religion. Child Development, 68, 484-95.

Helwig, C. 2006. The Development of Personal Autonomy Throughout Cultures. Cognitive Development, 21, 458-73.

Helwig, C. C. \& McNeil, J. 2011. The Development of Conceptions of Personal Autonomy, Rights, and Democracy and Their Relation to Psychological Well-Being. In V. Chirkov, R. Ryan, \& K. Sheldon (eds) Personal Autonomy in Cultural Contexts: Global erspectives on the Psychology of Agency, Freedom, and People's Well-Being (pp. 24156). New York: Springer.

Helwig, C. C., To, S., Wang, Q., Liu, C., \& Yang, S. 2014. Judgments and Reasoning About Parental Discipline Involving Induction and Psychological Control in China and Canada. Child Development, 85, 1150-67.

Howe, R. B., \& Covell, K. 2005. Empowering Children: Children's Rights Education As a Pathway to Citizenship. Toronto: University of Toronto Press. 
Jia, Y., Way, N., Ling, G., Yoshikawa, H., Chen., X., Hughes, D., Ke, Xiaoyan, \& Lu, Zuhong. 2009. The Influence of Student Perceptions of School Climate on Socioemotional and Academic Adjustment: A comparison of Chinese and American Adolescents. Child Development, 80, 1514-30.

Joussemet, M., Mageau, G. A., \& Koestner, R. 2014. Promoting Optimal Parenting and Children's Mental Health: A Preliminary Evaluation of the How-To Parenting Program. Journal of Child and Family Studies, 23, 949-64.

Khoury-Kassabri, M., \& Ben-Arieh, A. 2009. School Climate and Children's Views of Their Rights: A multi-Cultural Perspective Among Jewish and Arab Adolescents. Children and Youth Services Review, 31, 97-103.

Kovacs, M. 2011. Children's Depressive Inventory (2nd ed.) (CDI-2) Manual. North Tonawanda, NY: Multi-Health Systems.

Kuczynski, L., \& Knafo, A. 2014. Innovation and Continuity in Socialization, Internalization, and Acculturation. In M. Killen \& J. G. Smetana (eds) Handbook of moral development (2nd Edition, pp. 93-112). New York: Psychology Press.

Lahat, A., Helwig, C. C., Yang, S., Tan, D., \& Liu, C. 2009. Mainland Chinese Adolescents' Judgments and Reasoning About Self-Determination and Nurturance Rights. Social Development, 18, 690-710.

Lo, C., Helwig, C. C., Chen, S. X., Ohashi, M. M., \& Cheng, C. M. 2011. A Needs-Based Perspective on Cultural Differences in Identity Formation. Identity, 11, 211-30.

Mackerras, C. 2001. The New Cambridge Handbook of Contemporary China. Cambridge, England: Cambridge University Press.

Markus, H. R., \& Kitayama, S. 1991. Culture and the self: Implications for cognition, emotion, and motivation. Psychological Review, 98, 224-53.

Marsiglio, W., Amato, P., Day, R. D., \& Lamb, M. E. 2000. Scholarship on Fatherhood in the 1990s and Beyond. Journal of Marriage and the Family, 62, 1173-91.

Melton, G. B. 1980. Children's Concepts of Their Rights. Journal of Clinical Child Psychology, 9, 186-90.

Naftali, O. 2009. Empowering the Child: Children's Rights, Citizenship, and the State in Contemporary China. The China Journal, 61, 79-103.

Nucci, L. 2001. Education in the Moral Domain. Cambridge, UK: Cambridge University Press.

Nucci, L. P., Camino, C., \& Sapiro, C. 1996. Social Class Effects on Northeastern Brazilian Children's Conceptions of Areas of Personal Choice and Social Regulation. Child Development, 67, 1223-42.

Nussbaum, M. 2000. Women and Human Development: The Capabilities Approach. Cambridge: Cambridge University Press.

Pandey, S., \& Elliot, W. 2010. Suppressor Variables in Social Work Research: Ways to Identify in Multiple Regression Models. Journal of the Society for Social Work and Research, 1, 28-40.

Peterson-Badali, M., Morine, S., Ruck, M. D., \& Slonim, N. 2004. Predictors of Maternal and Child Attitudes towards Children's Nurturance and Self-Determination Rights. Journal of Early Adolescence, 24, 159-79.

Peterson-Badali, M., Ruck, M. D., \& Ridley, E. 2003. College Students' Attitudes toward Children's Nurturance and Self-Determination Rights. Journal of Applied Social Psychology, 33, 730-55.

Piaget, J. 1932. The Moral Judgment of the Child. London: Routledge \& Kegan Paul.

Qin, L., Pomerantz, E. M, \& Wang, Q. 2009. Are Gains in Decision-Making Autonomy During Early Adolescence Beneficial for Emotional Functioning? The Case of the United States and China. Child Development, 80, 1705-21.

Reynolds, C. R. \& Richmond, B. 0. 2008. Revised Children's Manifest Anxiety Scale (2 ${ }^{\text {nd }}$ ed.) (RCMAS-2): Manual. Los Angeles, CA: Western Psychological Services.

Richardson, H. S. 2002. Democratic Autonomy: Public Reasoning About the Ends of Policy. New York: Oxford University Press. 
Robbins, R. J. 1995. An Assessment of Perceived Autonomy-Support and Control: Child and Parent Correlates. Unpublished doctoral dissertation, University of Rochester, Rochester, NY.

Rogers, C. M. \& Wrightsman, L. S. 1978. Attitudes towards Children's Rights: Nurturance or Self-Determination? Journal of Social Issues, 34, 59-68.

Ruck, M. D., Abramovitch, R., \& Keating, D. P. 1998. Children's and Adolescents' Understanding of Rights: Balancing Nurturance and Self-Determination. Child Development, 69, 404-17.

Ruck, M. D., Tenenbaum, H., \& Willenberg, I. 2011. South African Mixed-Race Children's and Mothers' judgments and Reasoning about Nurturance and self-determination Rights. Social Development, 20, 431-643.

Sen, A. 2009. The idea of justice. Cambridge, MA: Harvard University Press.

Smetana, J. G. 2011. Adolescents, Families, and Social Development: How Teens Construct Their Worlds. Malden, MA: Wiley-Blackwell.

Terry, T. \& Huebner, E. S. 1995. The Relationship Between Self-Concept and Life Satisfaction in Children. Social Indicators Research, 35, 39-52.

Torney, J., Oppenheim, A., \& Farnen, R. 1975. Civic Education in Ten Countries: An Empirical Study. New York: Wiley.

Torney-Purta, J., Lehmann, R., Oswald, H., \& Schultz, W. 2001. Citizenship and Education in Twenty-Eight Countries: Civic Knowledge and Engagement at Age Fourteen. Amsterdam: IEA.

Turiel, E. 2006. The Development of Morality. In W. Damon and R. Lerner (Series eds) \& N. Eisenberg (vol. ed.), Handbook of Child Psychology. Vol. 3: Social, Emotional, and Personality Development (6 ${ }^{\text {th }}$ ed., pp. 789-857). New York: Wiley.

United Nations General Assembly. 1989. United Nations Convention on the Eights of the Child. New York: UNICEF.

Vansteenkiste, M., Soenens, B., Van Petegem, S., \& Duriez, B. 2014. Longitudinal Associations Between Adolescent Perceived Degree and Style of Parental Prohibition and Internalization and Defiance. Developmental Psychology, 50, 22936.

Vansteenkiste, M., Zhou, M., Lens, W., \& Soenens, B. 2005. Experiences of Autonomy and Control Among Chinese Learners: Vitalizing or Immobilizing? Journal of Educational Psychology, 97, 468-83.

Vieno, A., Perkins, D. D., Smith, T. M., \& Santinello, M. 2005. Democratic School Climate and Sense of Community in School: A Multilevel Analysis. American Journal of Community Psychology, 36, 327-41.

Way, N., Reddy, R., \& Rhodes, J. 2007. Students' Perceptions of School Climate During the Middle School Years: Associations with Trajectories of Psychological and Behavioral adjustment. American Journal of Community Psychology, 40, 194-213.

Wentzel, K. R. 1997. Student Motivation in Middle School: The Role of Perceived Pedagogical Caring. Journal of Educational Psychology, 89, 411-19.

Yang, B., Ollendick, T. H., Dong, Q., Xia, Y., \& Lin, L. 1995. Only Children and Children with Siblings in the People's Republic of China: Levels of Fear, Anxiety, and Depression. Child Development, 66, 1301-11.

Zhang, W. \& Fuligni, A. J. 2006. Authority, Autonomy, and Family Relationships Among Adolescents in Urban and Rural China. Journal of Research on Adolescence, 16, 52737.

Zhang, X., Zheng, X., \& Wang, L. 2003. Comparative Research on Individual Modernity of Adolescents Between Town and Countryside in China. Asian Journal of Social Psychology, 6, 61-73. 
Shaogang Yang

(Guangdong University of Foreign Studies, 200611497@oamail.gdufs.edu.cn)

Sharon To and Charles C. Helwig

(University of Toronto, sharonto@gmail.com, helwig@psych.utoronto.ca)

Chinese Adolescents' Moral Reasoning of Rights Attitudes and Psychological Well-Being

Abstract. This study examined rural and urban Chinese adolescents' (aged 13-19 years, $\mathrm{N}=395$ ) attitudes toward children's self-determination and nurturance rights, and how these attitudes relate to various dimensions of socialization in their family and school environments, including perceptions of parental and teacher autonomy support and responsiveness and family and school democratic climate. Relations between these variables and psychological well-being also were examined. Perceived parent and teacher autonomy support and responsiveness and democratic climate differentially predicted attitudes toward each type of right and were positively correlated with adolescents' psychological well-being. Our findings suggest that environments that are structured more democratically and that are responsive to children's autonomy needs contribute to their psychological health and well-being in diverse cultural settings.

Keywords: Chinese adolescents; self-determination right; nurturance right; psychological well-being

Ethics in Progress (ISSN 2084-9257). Vol. 8 (2017). No. 1, Art. \#17, pp. 263-278.

Creative Commons BY-SA 3.0

Doi: 10.14746/eip.2017.1.17 